\title{
Positive Blood Culture
}

National Cancer Institute

\section{Source}

National Cancer Institute. Positive Blood Culture. NCI Thesaurus. Code C122437.

A laboratory test result demonstrating the presence of a specific organism in a blood sample after culture. 